\title{
Geometric Lagrangian averaged Euler-Boussinesq and primitive equations
}

\author{
Gualtiero Badin \\ Center for Earth System Research and Sustainability (CEN), University of Hamburg, \\ D-20146 Hamburg, Germany \\ E-mail: gualtiero.badin@uni-hamburg.de
}

Marcel Oliver

School of Engineering and Science, Jacobs University, D-28759 Bremen, Germany

E-mail: oliver@member.ams.org

\section{Sergiy Vasylkevych}

Center for Earth System Research and Sustainability (CEN), University of Hamburg, D-20146 Hamburg, Germany

E-mail: sergiy.vasylkevych@uni-hamburg.de

11 September 2018

\begin{abstract}
In this article we derive the equations for a rotating stratified fluid governed by inviscid Euler-Boussinesq and primitive equations that account for the effects of the perturbations upon the mean. Our method is based on the concept of geometric generalized Lagrangian mean recently introduced by Gilbert and Vanneste, combined with generalized Taylor and horizontal isotropy of fluctuations as turbulent closure hypotheses. The models we obtain arise as Euler-Poincaré equations and inherit from their parent systems conservation laws for energy and potential vorticity. They are structurally and geometrically similar to Euler-Boussinesq- $\alpha$ and primitive equations- $\alpha$ models, however feature a different regularizing second order operator.
\end{abstract}

Keywords: Lagrangian averaging, stratified geophysical flows, turbulence, EulerPoincaré equations

\section{Introduction}

The goal of this article is to derive a self-consistent system of equations for the flow of an inviscid stratified fluid that accounts for the effects of the perturbations upon the mean. To this end, each realization of the flow is decomposed into the mean part and a small fluctuation. The effects of the fluctuations on the mean flow is then described by an appropriate mean flow theory. 
The definition of mean flow is subject to choice and can be approached either from Eulerian or Lagrangian point of view (e.g. [5, 9]). Eulerian mean theories define the mean flow by averaging fluid velocities at each spatial location. Lagrangian averaging describes the mean and deviations in terms of particle trajectories. Since most conservation laws in fluids hold for each fluid parcel, the Lagrangian point of view is more natural whenever the conservation laws are emphasized and this is the path that we pursue in the article.

The equations for the mean flow will be expressed in the form of $\alpha$-models for turbulence, which will be derived making use of geometric Lagrangian averaging. The original $\alpha$-models $[15,16]$ rely on the observation that replacing the kinetic energy with its $H^{1}$ counterpart

$$
\int_{M}|\boldsymbol{u}|^{2} \mathrm{~d} \boldsymbol{x} \mapsto \int_{M}|\boldsymbol{u}|^{2}+\alpha^{2}|\nabla \boldsymbol{u}|^{2} \mathrm{~d} \boldsymbol{x}
$$

in the fluid Lagrangian results in filtering, which moderates the growth of instabilities at wave numbers larger than $O\left(1 / \alpha^{2}\right)$, has a negligible impact on large scales and geophysical balances, and preserves the Hamiltonian structure of the equations and accompanying conservation laws. Motivated by this observation, a number of turbulence models were proposed, including among others, Euler- $\alpha$, Navier-Stokes- $\alpha$ (NS- $\alpha$ ), EulerBoussinesq- $\alpha$ (EB- $\alpha$ ), and primitive equations- $\alpha$ (PE- $\alpha)[7,15,16,12,13,21]$. These models were originally derived as abstract Euler-Poincaré equations arising from the above regularization in the appropriate context. Later on Euler- $\alpha$ and NS- $\alpha$ were rederived via Lagrangian averaging, further justifying their use for turbulence modelling.

The concept of Lagrangian averaging was introduced by Andrews and McIntyre in their seminal paper [2] (see also e.g. [3, 6, 26, 27, 32, 28, 29, 33, 34, 35]). According to the generalized Lagrangian mean (GLM) theory of Andrews and McIntyre, the mean flow is defined by Reynolds averaging particle positions in Eucledean space for each Lagrangian label $\boldsymbol{a}$, i.e.

$$
\boldsymbol{\eta}(\boldsymbol{a}, t) \equiv\left\langle\boldsymbol{\eta}^{\xi}(\boldsymbol{a}, t)\right\rangle,
$$

where $\boldsymbol{\eta}$ is the Lagrangian mean flow, $\left\{\eta^{\xi}\right\}$ is the ensemble of the flows to average, and $\langle\cdot\rangle$ is the ensemble average. The mean Lagrangian velocity $\boldsymbol{u}$ is then determined from the mean flow by time differentiation

$$
\boldsymbol{u}(\boldsymbol{x}, t)=\frac{\partial}{\partial t} \boldsymbol{\eta}(\boldsymbol{a}, t) \quad \text { with } \quad \boldsymbol{a}=\boldsymbol{\eta}^{-1}(\boldsymbol{x}, t) .
$$

In order to obtain a closed system of equations for the mean, one must supplement the GLM equations with closure laws governing the evolution of fluctuations.

A number of models for turbulent flow, including among others, Holm's Lagrangian Averaged Euler (LAE), Lagrangian mean Euler-Boussinesq (LMEB) and Lagrangian mean motion (LMM) equations, Euler- $\alpha$, and Navier-Stokes- $\alpha$ (NS- $\alpha$ ) EB- $\alpha$ and PE$\alpha[7,15,11,12,13,21]$, were derived combining GLM with the generalized Taylor hypothesis, which postulates that the fluctuations vector field $\boldsymbol{\xi} \equiv \boldsymbol{\eta}^{\boldsymbol{\xi}}-\boldsymbol{\eta}$ is Lie transported by the mean flow, i.e.

$$
\partial_{t} \boldsymbol{\xi}+\nabla_{\boldsymbol{u}} \boldsymbol{\xi}-\nabla_{\boldsymbol{\xi}} \boldsymbol{u}=0,
$$


where we employ the geometric notation $\nabla_{\boldsymbol{u}} \boldsymbol{\xi}$ to express the derivative of a field $\boldsymbol{\xi}$ in the direction of $\boldsymbol{u}$, so that $\nabla_{\boldsymbol{u}} \boldsymbol{\xi} \equiv(\boldsymbol{u} \cdot \nabla) \boldsymbol{\xi}$.

The major drawback of the original GLM theory is its reliance on Euclidean space structure to define the mean flow. Not only it prevents a seamless transfer of the theory to the manifold context, but also potentially breaks the incompressibility of the mean even if all the averaged flows are incompressible. This poses an unsatisfying dilemma: one either has to accept small compressibility of order $O\left(|\boldsymbol{\xi}|^{2}\right)$ or impose additional ad hoc constraints on second order fluctuations, which restore incompressibility. The first approach is taken in [11], while the latter was used in [21] to derive the Euler- $\alpha$ model.

Recently, Gilbert and Vanneste [10] proposed an alternative method of Lagrangian averaging that accounts for the underlying geometry. In the resulting geometric GLM (GGLM), rather than averaging particle trajectories, one averages the flows themselves, relying on the manifold structure of a diffeomorphism group in doing so. More precisely, the GGLM mean flow is defined as the Riemannian centre of mass on an appropriately chosen diffeomorphism group $\mathcal{D}$,

$$
\boldsymbol{\eta}=\underset{\Psi \in \mathcal{D}}{\arg \min }\left\langle d^{2}\left(\Psi, \boldsymbol{\eta}^{\boldsymbol{\xi}}\right)\right\rangle,
$$

where $d$ is the geodesic distance on $\mathcal{D}$. Then, the fluctuations, rather than being vector fields, are the diffeomorphisms connecting individual realizations with the mean, so that

$$
\boldsymbol{\eta}^{\xi}=\boldsymbol{\xi} \circ \boldsymbol{\eta}
$$

We remark that if one chooses a volume preserving diffeorphism group $\mathcal{D}_{\mu}$ as a configuration space, the mean flow is automatically volume preserving.

Another important choice arising in turbulence modelling is the treatment of Taylor diffusivity tensor $\kappa=\langle\boldsymbol{\xi} \otimes \boldsymbol{\xi}\rangle$. An attractive property is isotropy,

$$
\langle\boldsymbol{\xi} \otimes \boldsymbol{\xi}\rangle=\alpha I \text {, }
$$

where $I$ is the identity matrix, as it significantly simplifies the equations of motion, leading, among others, to classical Euler- and NS- $\alpha$ equations. Combining GGLM with Taylor's hypothesis and isotropy yields the Euler- $\alpha$, Camassa-Holm and EPdiff equations $[22,24]$ as Euler's and Burgers' mean flow, respectively. An alternative approach is to study a coupled dynamical system for the mean velocity and Taylor diffusivity tensor, where the issue of proper initialization for $\kappa$ is a significant one. The examples of this approach are LMEB and anisotropic Euler- $\alpha$ equations [11, 21].

The stratified analogues of the classical Euler- $\alpha$ equations, the Euler-Bousinessq- $\alpha$ $(\mathrm{EB}-\alpha)$ and primitive equations- $\alpha(\mathrm{PE}-\alpha)$ were, to the best of our knowledge, never derived as a turbulence model, although they were proposed and studied numerically $[15,16,18]$. This is most likely due to the fact that isotropy (7) is not compatible with rigid lid boundary conditions, natural for ideal geophysical flows. We overcome this issue by noting that rigid lid boundary conditions are compatible with horizontal isotropy 


$$
\kappa=\left(\begin{array}{lll}
1 & 0 & 0 \\
0 & 1 & 0 \\
0 & 0 & 0
\end{array}\right)
$$

on a horizontal strip. Notice that in the atmosphere and in the ocean, the term "horizontal" indicates a flow either along geopotential or isopycnal surfaces, respectively. In GGLM, horizontal isotropy must be interpreted accordingly.

Complimenting GGLM with the generalized Taylor hypothesis and horizontal isotropy as closure assumptions, we derive the models for the mean flow governed by the inviscid Euler-Boussinesq and primitive equations. We will call the respective models the horizontally isotropic Lagrangian averaged Euler-Boussinesq (HILAEB) and the horizontally isotropic Lagrangian averaged primitive equations (HILAPE). HILAEB and HILAPE are structurally and geometrically similar to EB- $\alpha$ and PE- $\alpha$, in particular they arise as Euler-Poincaré equations on the diffeomorphism group, and inherit conservation laws from their respective parent systems. However, we replace the full 3D Laplacian in $\mathrm{EB}-\alpha$ and PE- $\alpha$ by a horizontal one,

$$
\Delta_{h}=\partial_{x_{1} x_{1}}+\partial_{x_{2} x_{2}} .
$$

The discussion whether $\alpha$-models are good turbulence models (see, for instance, $[1,14,20])$, is beyond the scope of the present article. Instead, the derivation exposes a set of assumptions, sufficient to justify the use of the models.

The article has the following structure. In section 2 we introduce inviscid EulerBoussinesq, primitive equations, and the notation to be used throughout the article. In section 3 we review the GGLM concept and describe our method in a general setting. Section 4 is devoted to the closure hypothesis. In section 5 we recall the variational principles for EB and PE, followed by derivation of HILABE in section 7 and HILAPE in section 8, respectively. We conclude by the discussion of results is section 9 .

\section{Inviscid rotating Euler-Boussinesq and primitive equations}

Throughout the paper, we adapt the following notation: bold-face letters always denote three-component objects, while regular typeface is used for their horizontal parts; e.g., we write $\boldsymbol{u}=\left(u_{1}, u_{2}, u_{3}\right)^{T}=\left(u, u_{3}\right)^{T}$ for the full three-dimensional fluid velocity, $\boldsymbol{x}=\left(x_{1}, x_{2}, z\right)^{T}=(x, z)^{T}$, for spatial coordinate, $\boldsymbol{a}=\left(a_{1}, a_{2}, a_{3}\right)$ for particle labels, and $\nabla=\left(\partial_{x_{1}}, \partial_{x_{2}}, \partial_{z}\right)=\left(\nabla, \partial_{z}\right)$ for gradients. Further, we write $u^{\perp}=\left(-u_{2}, u_{1}\right)^{T}$ to denote the counter clockwise rotation of $u$ by $\pi / 2, \boldsymbol{u}_{h}=(u, 0)^{T}$ the projection of $\boldsymbol{u}$ onto the horizontal coordinate plane, and $\boldsymbol{u}^{\perp}=\boldsymbol{e}_{z} \times \boldsymbol{u}=\left(u^{\perp}, 0\right)^{T}$. We will also write $\Delta_{h} \equiv \partial_{x_{1} x_{1}}+\partial_{x_{2} x_{2}}$ for the horizontal Laplacian to distinguish it from full 3-D Laplace operator $\Delta \equiv \Delta_{h}+\partial_{z z}$.

With such notation in place, the inviscid Euler-Boussinesq equations in a rotating 
reference frame then read

$$
\begin{aligned}
& \partial_{t} \boldsymbol{u}+\nabla_{\boldsymbol{u}} \boldsymbol{u}+\boldsymbol{\Omega} \times \boldsymbol{u}+\theta \boldsymbol{e}_{z}+\nabla p=0 \\
& \partial_{t} \theta+\nabla_{\boldsymbol{u}} \theta=0, \\
& \nabla \cdot \boldsymbol{u}=0
\end{aligned}
$$

where $\boldsymbol{\Omega}(x) / 2$ is the local angular velocity vector. The EB equations approximate the motion of stratified inviscid incompressible Newtonian fluid by neglecting the variations in fluid density everywhere except in the gravity term. They are physically relevant for large- and mesoscale flows both in atmosphere and ocean. In the former case, $z, p$ and $\theta$ stand for pressure, geopotential and potential temperature, while in the context of ocean flows they represent depth, pressure and buoyancy, respectively.

For simplicity, we consider equations in the strip $M=M^{\prime} \times[0, H]$ of constant height $H$ with no mass flux conditions

$$
u_{3}=0 \text { for } z=0 \text { and } z=H
$$

on vertical boundaries. The horizontal projection of the strip, $M^{\prime}$, is assumed to be either a plane, $M^{\prime}=\mathbb{R}^{2}$, a double periodic domain $M^{\prime}=\mathbb{T}^{2}$, or an infinite cylinder $M^{\prime}=\mathbb{R} \times \mathbb{T}$. In all cases we assume sufficient decay at infinity, so that volume integrals defining the Lagrangians below are finite and we can freely integrate by parts in the horizontal variables without incurring boundary terms.

The primitive equations are a further simplification of the EB system that arises by neglecting the vertical component of the Coriolis force and imposing hydrostatic balance in the vertical momentum equation. The form of the primitive equations considered here reads

$$
\begin{aligned}
& \partial_{t} \boldsymbol{u}_{h}+\nabla_{\boldsymbol{u}} \boldsymbol{u}_{h}+f \boldsymbol{u}^{\perp}+\theta \boldsymbol{e}_{z}+\nabla p=0, \\
& \partial_{t} \theta+\nabla_{\boldsymbol{u}} \theta=0, \\
& \nabla \cdot \boldsymbol{u}=0,
\end{aligned}
$$

where $f=f(x)$ is the Coriolis frequency.

\section{Turbulence models via Lagrangian averaging for Hamiltonian systems}

This section describes the general procedure we use to derive turbulence models for flows governed by EB and primitive equations. The derivation itself is postponed to section 6. The procedure is applicable to arbitrary Hamiltonian systems on diffeomorphism groups. We refer the reader to $[22,24]$ for further examples of such applications.

The cornerstone of our approach is the concept of geometric generalized Lagrangian

mean introduced by Gilbert and Vanneste [10]. Suppose $\{\beta\}$ is an arbitrary index set, $\left\{\boldsymbol{\eta}_{\beta}\right\}$ is an ensemble of flows parametrized by $\beta$ and $\left\langle f_{\beta}\right\rangle$ denotes the average of scalar quantity $f$ over $\{\beta\}$. The precise way in which this averaging is defined does not matter as long as it commutes with time-differentiation and spatial integration

$$
\left\langle\int_{M} f_{\beta}(x) \mathrm{d} \boldsymbol{x}\right\rangle=\int_{M}\left\langle f_{\beta}(x)\right\rangle \mathrm{d} \boldsymbol{x}
$$


and the chosen closure assumptions are satisfied with respect to the induced notion of the Lagrangian mean flow. The GGLM exploits the fact that flow maps $\eta_{\beta}$ are points on an infinite dimensional Riemannian manifold, namely an appropriately chosen diffeomorphism group, and therefore their ensemble average can be defined as the geometric centre of mass on that group.

To make this idea more precise, we choose as the configuration space $\mathcal{D}=\mathcal{D}_{\mu}^{s}(M)$ the group of volume and orientation preserving diffeomorphisms of Sobolev class $s>5 / 2$ which leave the boundary invariant,

$$
\mathcal{D}_{\mu}^{s}(M) \equiv\left\{\boldsymbol{\eta} \in H^{s}(M) \mid \boldsymbol{\eta}^{-1} \in H^{s}(M), \boldsymbol{\eta}(\partial M)=\partial M, \operatorname{det} \nabla \boldsymbol{\eta}=1\right\},
$$

Let $\mathfrak{X}_{\text {div }} \equiv \mathfrak{X}_{\text {div }}^{s}(M)$ be the space of $H^{s}$ divergence free vector fields on $M$. Then, following $[25,8], \mathcal{D}_{\mu}^{s}$ is a smooth infinite dimensional manifold with tangent bundle

$$
T_{\boldsymbol{\eta}} \mathcal{D}_{\mu}^{s}(M)=\left\{\boldsymbol{u} \circ \boldsymbol{\eta} \mid \boldsymbol{u} \in \mathfrak{X}_{\mathrm{div}}^{s}(M), u_{3}=0 \text { on } \partial M\right\} .
$$

Equipping $T_{\boldsymbol{\eta}} \mathcal{D}$ with $L^{2}$ inner product

$$
(\boldsymbol{v} \circ \boldsymbol{\eta}, \boldsymbol{w} \circ \boldsymbol{\eta})=\int_{M} \boldsymbol{v} \cdot \boldsymbol{w} \mathrm{d} \boldsymbol{x} \quad \text { for all } \boldsymbol{v}, \boldsymbol{w} \in \mathfrak{X}
$$

turns $\mathcal{D}$ into a weak Riemmanian manifold. Then, one can define the geodesic distance between maps $\boldsymbol{\psi}, \boldsymbol{\phi} \in \mathcal{D}$ via

$$
d^{2}(\boldsymbol{\phi}, \boldsymbol{\psi})=\inf _{\substack{\gamma_{s}:[0,1] \rightarrow \mathcal{D} \\ \gamma_{0}=\phi, \gamma_{1}=\boldsymbol{\psi}}} \int_{0}^{1}\left(\boldsymbol{\gamma}_{s}^{\prime}, \boldsymbol{\gamma}_{s}^{\prime}\right) \mathrm{d} t
$$

where $\gamma_{s}$ is a geodesic in $\mathcal{D}$ connecting $\boldsymbol{\phi}$ and $\boldsymbol{\psi}$ with prime here and further on denoting derivative with respect to fictitious time $s$ parametrizing the geodesics.

We remark that $\mathcal{D}$ is not geodesically complete with respect to the $L^{2}$ metric, therefore the above definition is only a formal one. Nevertheless, in case when $M$ is compact, $\mathcal{D}$ has a well-defined exponential map [8], so that (17) is defined in a neighbourhood of the identity and, by right invariance of the metric, in the neighbourhood of any $\boldsymbol{\eta} \in \mathcal{D}$.

Now we note that if $\left\{\boldsymbol{\eta}_{\beta}\right\}$ is an ensemble of sufficiently smooth EB or PE flows with no-mass-flux boundary conditions, then, necessarily, $\left\{\boldsymbol{\eta}_{\beta}\right\} \subset \mathcal{D}$. Following Gilbert and Vanneste, the Lagrangian mean flow $\left\langle\boldsymbol{\eta}_{\beta}\right\rangle_{L}$ is defined as the ensemble's geometric centre of mass in the volumorphisms group, i.e.

$$
\boldsymbol{\eta}=\left\langle\boldsymbol{\eta}_{\beta}\right\rangle_{L} \equiv \underset{\boldsymbol{\Psi} \in \mathcal{D}}{\arg \min }\left\langle d^{2}\left(\boldsymbol{\Psi}, \boldsymbol{\eta}_{\beta}\right)\right\rangle .
$$

Then a fluctuation is defined as a diffeomorphism $\boldsymbol{\xi}_{\beta}$ connecting the mean with the corresponding realization, so that

$$
\boldsymbol{\eta}_{\beta}=\boldsymbol{\xi}_{\beta} \circ \boldsymbol{\eta} .
$$

The Lagrangian mean velocity field $\boldsymbol{u}$ is recovered by differentiating the mean flow in time, i.e.

$$
\dot{\boldsymbol{\eta}} \equiv \partial_{t} \boldsymbol{\eta}(\boldsymbol{a}, t)=\boldsymbol{u} \circ \boldsymbol{\eta}(\boldsymbol{a}, t)
$$


This definition has three advantages. First, it is geometrically intrinsic. Second, the mean flow automatically satisfies no-mass-flux boundary conditions and is incompressible by virtue of being a member of $\mathcal{D}$. Third, for any set of quantities $\left\{q_{\beta}\right\}$ that are materially conserved by the respective flows $\boldsymbol{\eta}_{\beta}$, their Lagrangian average

$$
\bar{q}^{L}=\left\langle\boldsymbol{\xi}_{\beta}^{*} q_{\beta}\right\rangle,
$$

where the star denotes the pull-back operation, is materially conserved by the mean flow $\boldsymbol{\eta}$. In particular, the mean motion will have a well-defined potential vorticity (PV) conservation law, provided each of the averaged flows has one. As usual, to obtain a turbulence model, one needs to supply closure assumptions on statistical properties and on the evolution of fluctuations that result in a closed system of equations for the mean flow.

The drawback of the above construction is that the equations governing the Lagrangian mean velocity $\boldsymbol{u}$ is difficult to write explicitly. For Hamiltonian systems this difficulty can be overcome by combining the GGLM concept with averaging of the Lagrangian, as is done in [21]. Consider a Hamiltonian system on $\mathcal{D}$ defined by a Lagrangian $L$, i.e., the system whose flows are stationary points of the action

$$
S=\int_{0}^{t} L(\boldsymbol{\eta}, \dot{\boldsymbol{\eta}}) \mathrm{d} t, \quad \boldsymbol{\eta} \in \mathcal{D}
$$

with respect to variation of $\boldsymbol{\eta}$ fixed at the temporal end points. In section 5 we recall how the EB and primitive equations fit into this variational framework.

Let $\varepsilon>0$ be a fixed small parameter describing the amplitude of fluctuations, let $\boldsymbol{\eta}_{\varepsilon, \beta}$ be a single realization from an ensemble of turbulent flows of the system, and let $\boldsymbol{\eta}=\left\langle\boldsymbol{\eta}_{\varepsilon, \beta}\right\rangle_{L}$ be the Lagrangian mean of the ensemble in the GGLM sense. We expand $\left\langle L\left(\boldsymbol{\eta}_{\varepsilon, \beta}, \dot{\boldsymbol{\eta}}_{\varepsilon, \beta}\right)\right\rangle$ in powers of $\varepsilon$, expressing the result in terms of the mean flow and statistical properties of the fluctuations, and impose closure conditions such that

$$
\left\langle L\left(\boldsymbol{\eta}_{\varepsilon, \beta}, \dot{\boldsymbol{\eta}}_{\varepsilon, \beta}\right)\right\rangle=\bar{L}(\boldsymbol{\eta}, \dot{\boldsymbol{\eta}})+O\left(\varepsilon^{3}\right) .
$$

The averaged Lagrangian $\bar{L}$ then yields averaged model equations via the variational principle.

\section{Closure assumptions}

By construction, fluctuations $\boldsymbol{\xi}_{\varepsilon, \beta}$ are volumorphisms such that

$$
\boldsymbol{\eta}_{\varepsilon, \beta}=\boldsymbol{\xi}_{\varepsilon, \beta} \circ \boldsymbol{\eta}
$$

Let $\boldsymbol{\xi}_{s, \beta}$, where $0 \leq s \leq \varepsilon$ and $\boldsymbol{\xi}_{0, \beta}=\mathrm{id}$, be the geodesics in $\mathcal{D}$ connecting $\boldsymbol{\eta}$ with realizations $\boldsymbol{\eta}_{\varepsilon, \beta}$. Define the fluctuation vector fields $\boldsymbol{w}_{s, \beta}$ by

$$
\boldsymbol{\xi}_{s, \beta}^{\prime}=\boldsymbol{w}_{s, \beta} \circ \boldsymbol{\xi}_{s, \beta} .
$$

Gilbert and Vanneste [10] show that $\boldsymbol{w}_{s, \beta}$ satisfy the Euler equations in fictitious time $s$,

$$
\boldsymbol{w}_{s, \beta}^{\prime}+\nabla_{\boldsymbol{w}_{s, \beta}} \boldsymbol{w}_{s, \beta}+\nabla \phi_{s, \beta}=0
$$


together with a constraint on the initial condition

$$
\left\langle\boldsymbol{w}_{\beta}\right\rangle=0,
$$

where the absence of $s$ index means evaluation at $s=0$. The potentials $\phi_{s, \beta}$ in (26) are uniquely (up to a constant) determined by the requirement to maintain incompressibility and boundary conditions. By the Hodge decomposition, they satisfy Poisson's equation

$$
\Delta \phi_{s, \beta}=-\operatorname{div}\left(\nabla_{\boldsymbol{w}_{s, \beta}} \boldsymbol{w}_{s, \beta}\right) \quad \text { in } M
$$

with matching Neumann boundary conditions

$$
\frac{\partial \phi}{\partial z}=-\left(\nabla_{\boldsymbol{w}_{s, \beta}} \boldsymbol{w}_{s, \beta}\right) \cdot \boldsymbol{e}_{z} \quad \text { on } \partial M .
$$

As before, let $\boldsymbol{u}$ denote the Eulerian velocity generating the Lagrangian mean flow $\boldsymbol{\eta}$. Both for the Euler-Boussinesq and the primitive equations we choose the generalized Taylor hypothesis

$$
\dot{\boldsymbol{w}}_{\beta}+\mathcal{L}_{u} \boldsymbol{w}_{\beta}=0
$$

and horizontal isotropy of fluctuations,

$$
\left\langle\boldsymbol{w}_{\beta} \otimes \boldsymbol{w}_{\beta}\right\rangle=\left(\begin{array}{ccc}
1 & 0 & 0 \\
0 & 1 & 0 \\
0 & 0 & 0
\end{array}\right),
$$

as closure assumptions.

The generalized Taylor hypothesis, which implies that first order fluctuation vector fields are transported by the mean flow, is the usual assumption for derivation of Lagrangian turbulence models $[11,7,12,13,21,22]$. On domains without boundary, the Euler- $\alpha$ equations were derived using full isotropy of fluctuations,

$$
\left\langle\boldsymbol{w}_{\beta} \otimes \boldsymbol{w}_{\beta}\right\rangle=I .
$$

However, it has been noted already in [21] that (29) is incompatible with no-massflux boundary conditions, hence anisotropic Lagrangian averaged equations must be considered on domains with boundaries. While our methods allow for derivation of anisotropic equations as well, we note that the horizontal isotropy is consistent with boundary conditions considered here. We further note that a horizontal initial condition for the Euler equation (26a) generally results in a nearly horizontal flow map for $s \leq \varepsilon$. Therefore a horizontal vector field $\boldsymbol{w}_{\beta}$ generates nearly horizontal fluctuation $\boldsymbol{\xi}_{\varepsilon, \beta}$. As remarked earlier, in geophysical stratified flows the vertical scale of motion is typically significantly smaller than horizontal one, so that the horizontal isotropy assumption is reasonable from physical standpoint.

\section{Variational principles for Euler-Boussinesq and primitive equations}

In this section we recall how $\mathrm{EB}$ and $\mathrm{PE}$ arise from a variational principle on $\mathcal{D}$. Let $\boldsymbol{\eta}$ denote the flow of a time-dependent Eulerian velocity field $\boldsymbol{u}$, i.e.

$$
\dot{\boldsymbol{\eta}} \equiv \partial_{t} \boldsymbol{\eta}(\boldsymbol{a}, t)=\boldsymbol{u} \circ \boldsymbol{\eta}(\boldsymbol{a}, t) .
$$


with $\boldsymbol{\eta}(\cdot, 0)$ being an identity map. The advection equation (10b) is then equivalent to

$$
\theta \circ \boldsymbol{\eta}=\theta_{0},
$$

where $\theta_{0}$ is the initial potential temperature distribution. Let $\boldsymbol{R}$ be a vector potential for the angular velocity $\boldsymbol{\Omega}$ and consider the Lagrangian

$$
L(\boldsymbol{\eta}, \dot{\boldsymbol{\eta}})=\int_{M} \frac{1}{2}|\dot{\boldsymbol{\eta}}|^{2}+\boldsymbol{R} \circ \boldsymbol{\eta} \cdot \dot{\eta}-\theta_{0} \eta_{3} \mathrm{~d} \boldsymbol{a}
$$

One can show that $\boldsymbol{u}$ and $\theta$ satisfy EB system (10) if and only if the flow map $\boldsymbol{\eta}$ is a stationary point of the action,

$$
\delta S=0, \quad S=\int_{0}^{t} L(\boldsymbol{\eta}, \dot{\boldsymbol{\eta}}) \mathrm{d} t, \quad \boldsymbol{\eta} \in \mathcal{D},
$$

with respect to variations of $\boldsymbol{\eta}$ fixed at temporal end points.

Let $\mathfrak{X}=T_{\text {id }} \mathcal{D}$. Because of (15), we can write $\delta \boldsymbol{\eta}=\boldsymbol{w} \circ \boldsymbol{\eta}$, with $\boldsymbol{w} \in \mathfrak{X}$. Using the particle relabelling symmetry,

$$
L(\boldsymbol{\eta}, \dot{\boldsymbol{\eta}})=\ell(\boldsymbol{u}, \theta) \equiv \int_{M} \frac{1}{2}|\boldsymbol{u}|^{2}+\boldsymbol{R} \cdot \boldsymbol{u}-\theta z \mathrm{~d} \boldsymbol{x},
$$

where, as always, $\boldsymbol{u}, \theta$, and $\boldsymbol{\eta}$ are related by (30)-(31), the variational principle leading to the EB equations can be restated in purely Eulerian terms as

$$
\delta \int_{0}^{t} \ell(\boldsymbol{u}, \theta) \mathrm{d} t=0
$$

subject to variations in $\boldsymbol{u}$ and $\theta$ obeying the Lin constraints

$$
\begin{aligned}
& \delta \boldsymbol{u}=\dot{\boldsymbol{w}}+[\boldsymbol{u}, \boldsymbol{w}], \\
& \delta \theta+\nabla_{\boldsymbol{w}} \theta=0 .
\end{aligned}
$$

Lin constraints reflect the fact that even though the reduced Lagrangian $\ell$ depends only on Eulerian quantities, the variations are still taken with respect to the flow map $\boldsymbol{\eta}$.

The Euler-Poincaré theorem for continua [15, 17] states that Euler-Poincaré equations for (33) or, equivalently, for (35), (36), are given by

$$
\int_{M}\left(\partial_{t}+\mathcal{L}_{\boldsymbol{u}}\right) \boldsymbol{m} \cdot \boldsymbol{w}+\frac{\delta \ell}{\delta \theta} \mathcal{L}_{\boldsymbol{w}} \theta \mathrm{d} \boldsymbol{x}=0 \text { for all } \boldsymbol{w} \in \mathfrak{X},
$$

where $\mathcal{L}$ denotes the Lie derivative and $\boldsymbol{m}$ is the momentum one-form $\boldsymbol{m}=\frac{\delta \ell}{\delta \boldsymbol{u}}$. Translated to the language of vector calculus, up to a vanishing term $\int_{M} \nabla\left(\boldsymbol{m}^{\sharp} \cdot \boldsymbol{u}\right) \cdot \boldsymbol{w} \mathrm{d} \boldsymbol{x}$, (37) reads

$$
\int_{M}\left(\partial_{t} \boldsymbol{m}^{\sharp}+\left(\nabla \times \boldsymbol{m}^{\sharp}\right) \times \boldsymbol{u}+\frac{\delta \ell}{\delta \theta} \nabla \theta\right) \cdot \boldsymbol{w} \mathrm{d} \boldsymbol{x}=0 \text { for all } \boldsymbol{w} \in \mathfrak{X},
$$

where $\boldsymbol{m}^{\sharp}$ is a vector field dual to $\boldsymbol{m}$ with respect to $L^{2}$ metric. Equation (38) expresses that the term in parenthesis is $L^{2}$-orthogonal to arbitrary sufficiently smooth divergencefree vector fields which are tangent to the boundary. The Hodge decomposition theorem then implies that this term must be a gradient. The EB momentum equation (10a) follows by noting that

$$
\boldsymbol{m}^{\sharp}=\boldsymbol{R}+\boldsymbol{u}, \quad \frac{\delta \ell}{\delta \theta}=-z,
$$


and using the vector identity

$$
(\nabla \times \boldsymbol{u}) \times \boldsymbol{u}=\nabla_{\boldsymbol{u}} \boldsymbol{u}-\frac{1}{2} \nabla|\boldsymbol{u}|^{2} .
$$

Analogous to EB system, the primitive equations arise from variational principle (33) on the diffeomorphism group $\mathcal{D}$ with Lagrangian

$$
L^{P}(\boldsymbol{\eta}, \dot{\boldsymbol{\eta}})=\int_{M} \frac{1}{2}|\dot{\eta}|^{2}+\boldsymbol{R} \circ \boldsymbol{\eta} \cdot \dot{\boldsymbol{\eta}}-\theta_{0} \eta_{3} \mathrm{~d} \boldsymbol{a},
$$

where $\boldsymbol{R}=(R(x), 0)^{T}$ is the vector potential for the Coriolis parameter, i.e. $\nabla \times \boldsymbol{R}=$ $\boldsymbol{\Omega}=f \boldsymbol{e}_{z}$

\section{Averaged EB and PE Lagrangians}

\subsection{Euler-Boussinesq}

Now we are ready to implement the program outlined in section 3 using the closure conditions (28). Using notation of section 4 , for $0 \leq s \leq \varepsilon$ define

$$
\boldsymbol{\eta}_{s, \beta}=\boldsymbol{\xi}_{s, \beta} \circ \boldsymbol{\eta}
$$

and let $\boldsymbol{u}_{s, \beta}$ and $\boldsymbol{u}$ denote Eulerian velocity fields generating $\boldsymbol{\eta}_{s, \beta}$ and $\boldsymbol{\eta}$, respectively, while $\theta_{s, \beta}$ and $\theta$ stand for corresponding potential temperature fields. We have

$$
\begin{array}{ll}
\dot{\boldsymbol{\eta}}_{s, \beta}=\boldsymbol{u}_{s, \beta} \circ \boldsymbol{\eta}_{s, \beta}, & \dot{\boldsymbol{\eta}}=\boldsymbol{u} \circ \boldsymbol{\eta}, \\
\theta_{s, \beta} \circ \boldsymbol{\eta}_{s, \beta}=\theta_{0}, & \theta \circ \boldsymbol{\eta}=\theta_{0} .
\end{array}
$$

Expand the velocity and potential temperature fields in powers of $s$,

$$
\begin{aligned}
& \boldsymbol{u}_{s, \beta}=\boldsymbol{u}+s \boldsymbol{u}_{\beta}^{\prime}+\frac{1}{2} s^{2} \boldsymbol{u}_{\beta}^{\prime \prime}+O\left(\varepsilon^{3}\right), \\
& \theta_{s, \beta}=\theta+s \theta_{\beta}^{\prime}+\frac{1}{2} s^{2} \theta_{\beta}^{\prime \prime}+O\left(\varepsilon^{3}\right),
\end{aligned}
$$

where we are using a uniform bound on the Taylor's series remainder, and substitute into EB Lagrangian to obtain

$$
\begin{aligned}
L_{\varepsilon} & \equiv\left\langle L\left(\boldsymbol{\eta}_{\varepsilon, \beta}, \dot{\boldsymbol{\eta}}_{\varepsilon, \beta}\right)\right\rangle \\
& =\frac{1}{2}\left\langle\int_{M}|\boldsymbol{u}|^{2}+2 \varepsilon \boldsymbol{u} \cdot \boldsymbol{u}_{\beta}^{\prime}+\varepsilon^{2}\left(\left|\boldsymbol{u}_{\beta}^{\prime}\right|^{2}+\boldsymbol{u} \cdot \boldsymbol{u}_{\beta}^{\prime \prime}\right) \mathrm{d} \boldsymbol{x}\right\rangle \\
& +\left\langle\int_{M} \boldsymbol{R} \cdot\left(\boldsymbol{u}+\varepsilon \boldsymbol{u}_{\beta}^{\prime}+\frac{\varepsilon^{2}}{2} \boldsymbol{u}_{\beta}^{\prime \prime}\right)-z\left(\theta+\varepsilon \theta_{\beta}^{\prime}+\frac{\varepsilon^{2}}{2} \theta_{\beta}^{\prime \prime}\right) \mathrm{d} \boldsymbol{x}\right\rangle+O\left(\varepsilon^{3}\right) .
\end{aligned}
$$

In order to obtain the mean flow Lagrangian in the closed form (23), one needs to

eliminate all quantities with primes from (45). The expansion fields $\boldsymbol{u}_{\beta}^{\prime}, \theta_{\beta}^{\prime}$, etc. can be expressed in terms of mean quantities and fluctuations by differentiating the curves at $s=0$. Indeed, the equality of partial derivatives

$$
\left(\dot{\boldsymbol{\eta}}_{s, \beta}\right)^{\prime}=\partial_{t}\left(\boldsymbol{\eta}_{s, \beta}^{\prime}\right)
$$

yields

$$
\left(\boldsymbol{u}_{s, \beta}^{\prime}+\nabla_{\boldsymbol{w}_{s, \beta}} \boldsymbol{u}_{s, \beta}\right) \circ \boldsymbol{\eta}_{s, \beta}=\left(\dot{\boldsymbol{w}}_{s, \beta}+\nabla_{\boldsymbol{u}_{s, \beta}} \boldsymbol{w}_{s, \beta}\right) \circ \boldsymbol{\eta}_{s, \beta}
$$


therefore, since $\boldsymbol{u}_{0, \beta}=\boldsymbol{u}$,

$$
\boldsymbol{u}_{\beta}^{\prime}=\dot{\boldsymbol{w}}_{\beta}+\mathcal{L}_{\boldsymbol{u}} \boldsymbol{w}_{\beta}
$$

Similarly, differentiating (31) yields

$$
\theta_{\beta}^{\prime}=-\nabla_{\boldsymbol{w}_{\beta}} \theta .
$$

The Taylor hypothesis (28a) implies

$$
\boldsymbol{u}_{\beta}^{\prime}=0
$$

and

$$
\begin{aligned}
\boldsymbol{u}_{\beta}^{\prime \prime} & =\dot{\boldsymbol{w}}_{\beta}^{\prime}+\mathcal{L}_{\boldsymbol{u}} \boldsymbol{w}_{\beta}^{\prime}+\mathcal{L}_{\boldsymbol{u}^{\prime}} \boldsymbol{w}_{\beta} \\
& =\dot{\boldsymbol{w}}_{\beta}^{\prime}+\mathcal{L}_{\boldsymbol{u}} \boldsymbol{w}_{\beta}^{\prime} .
\end{aligned}
$$

Differentiating (26a) in time and replacing $\dot{\boldsymbol{w}}_{\beta}$ by $-\mathcal{L}_{u} \boldsymbol{w}_{\beta}$ from (28a), we can express $\dot{\boldsymbol{w}}_{\beta}^{\prime}$ in terms of $\boldsymbol{w}_{\beta}, \boldsymbol{u}$, and $\phi$ to obtain

$$
\begin{aligned}
& \boldsymbol{u}_{\beta}^{\prime \prime}=\nabla_{\boldsymbol{w}_{\beta}}\left(\mathcal{L}_{\boldsymbol{u}} \boldsymbol{w}_{\beta}\right)+\nabla_{\mathcal{L}_{\boldsymbol{u}} \boldsymbol{w}_{\beta}} \boldsymbol{w}_{\beta}-\mathcal{L}_{\boldsymbol{u}}\left(\nabla_{\boldsymbol{w}_{\beta}} \boldsymbol{w}_{\beta}\right)-\mathcal{L}_{\boldsymbol{u}} \nabla \phi-\nabla \dot{\phi} \\
& =\nabla_{\nabla_{\boldsymbol{w}_{\beta}} \boldsymbol{w}_{\beta}} \boldsymbol{u}-\nabla_{\boldsymbol{w}_{\beta}} \nabla_{\boldsymbol{w}_{\beta}} \boldsymbol{u}-\mathcal{L}_{\boldsymbol{u}} \nabla \phi-\nabla \dot{\phi},
\end{aligned}
$$

where, in order to pass to the second line in (52), we used the standard vector identities

$$
\begin{aligned}
& \mathcal{L}_{\boldsymbol{u}} \boldsymbol{v}=\nabla_{u} \boldsymbol{v}-\nabla_{\boldsymbol{v}} \boldsymbol{u} \\
& \nabla_{\boldsymbol{w}} \nabla_{\boldsymbol{u}} \boldsymbol{v}-\nabla_{\boldsymbol{u}} \nabla_{\boldsymbol{w}} \boldsymbol{v}+\nabla_{\mathcal{L}_{\boldsymbol{u}} \boldsymbol{w}} \boldsymbol{v}=0
\end{aligned}
$$

for arbitrary vector fields $\boldsymbol{u}, \boldsymbol{v}$, and $\boldsymbol{w}$. We remark that the last identity holds since $M$ has no curvature. Finally, differentiating (49) and substituting $\boldsymbol{w}_{\beta}^{\prime}$ and $\theta_{\beta}^{\prime}$ from (26a) and (49), respectively, into the resulting expression yields

$$
\theta_{\beta}^{\prime \prime}=\nabla_{\nabla_{\boldsymbol{w}_{\beta}} \boldsymbol{w}_{\beta}} \theta+\nabla_{\nabla \phi_{\beta}} \theta+\nabla_{\boldsymbol{w}_{\beta}} \nabla_{\boldsymbol{w}_{\beta}} \theta
$$

Now, using (50), (49), (52), and (54), we can eliminate the higher order derivatives from (45) and express the azimuthally averaged Lagrangian $L_{\varepsilon}$ as

$$
\begin{aligned}
L_{\varepsilon} & =\ell(\boldsymbol{u}, \theta)+\varepsilon\left\langle\int_{M} z \nabla_{\boldsymbol{w}_{\beta}} \theta \mathrm{d} \boldsymbol{x}\right\rangle \\
& +\frac{\varepsilon^{2}}{2}\left\langle\int_{M}(\boldsymbol{u}+\boldsymbol{R}) \cdot\left(\nabla_{\nabla_{\boldsymbol{w}_{\beta}} \boldsymbol{w}_{\beta}} \boldsymbol{u}-\nabla_{\boldsymbol{w}_{\beta}} \nabla_{\boldsymbol{w}_{\beta}} \boldsymbol{u}-\mathcal{L}_{\boldsymbol{u}} \nabla \phi_{\beta}-\nabla \dot{\phi}_{\beta}\right) \mathrm{d} \boldsymbol{x}\right\rangle \\
& -\frac{\varepsilon^{2}}{2}\left\langle\int_{M} z\left(\nabla_{\nabla_{\boldsymbol{w}_{\beta}} \boldsymbol{w}_{\beta}} \theta+\nabla_{\nabla \phi_{\beta}} \theta+\nabla_{\boldsymbol{w}_{\beta}} \nabla_{\boldsymbol{w}_{\beta}} \theta\right) \mathrm{d} \boldsymbol{x}\right\rangle+O\left(\varepsilon^{3}\right) \\
& =\ell(\boldsymbol{u}, \theta)+\frac{\varepsilon^{2}}{2}\left\langle\int_{M}(\boldsymbol{u}+\boldsymbol{R}) \cdot\left(\nabla_{\nabla_{\boldsymbol{w}_{\beta}} \boldsymbol{w}_{\beta}} \boldsymbol{u}-\nabla_{\boldsymbol{w}_{\beta}} \nabla_{\boldsymbol{w}_{\beta}} \boldsymbol{u}-\mathcal{L}_{\boldsymbol{u}} \nabla \phi_{\beta}\right) \mathrm{d} \boldsymbol{x}\right\rangle \\
& -\frac{\varepsilon^{2}}{2}\left\langle\int_{M} z\left(\nabla_{\nabla_{\boldsymbol{w}_{\beta}} \boldsymbol{w}_{\beta}} \theta+\nabla_{\nabla \phi_{\beta}} \theta+\nabla_{\boldsymbol{w}_{\beta}} \nabla_{\boldsymbol{w}_{\beta}} \theta\right) \mathrm{d} \boldsymbol{x}\right\rangle+O\left(\varepsilon^{3}\right),
\end{aligned}
$$

where $\int_{M}(\boldsymbol{u}+\boldsymbol{R}) \cdot \nabla \dot{\phi}_{\beta} \mathrm{d} \boldsymbol{x}=0$ since divergence free fields are $L^{2}$-orthogonal to gradients while order $\varepsilon$ term vanishes due to $\left\langle\boldsymbol{w}_{\beta}\right\rangle=0$. 
We further simplify (55) by integrating by parts keeping in mind that $\boldsymbol{u}$ and $\boldsymbol{w}_{\beta}$ are divergence free, and using the horizontal isotropy of fluctuations. Let $\boldsymbol{v}$ denote an arbitrary (not necessarily divergence free) sufficiently smooth vector field. Then

$$
\begin{aligned}
\left\langle\int_{M} \nabla_{\nabla_{\boldsymbol{w}_{\beta}} \boldsymbol{w}_{\beta}} \boldsymbol{u} \cdot \boldsymbol{v} \mathrm{d} \boldsymbol{x}\right\rangle & =-\left\langle\int_{M} w_{\beta k} w_{\beta i} \partial_{x_{i}}\left(\frac{\partial u_{j}}{\partial x_{k}} v_{j}\right) \mathrm{d} \boldsymbol{x}\right\rangle \\
& =\int_{M} \partial_{x_{\alpha}}\left(\frac{\partial u_{j}}{\partial x_{\alpha}} v_{j}\right) \mathrm{d} \boldsymbol{x} \\
& =0,
\end{aligned}
$$

where we employ Einstein's convention on summing over repeated indices, and $\alpha$ denotes the index that runs over the horizontal components as opposed to Roman indices running through all three spatial coordinates. We also note that the horizontal isotropy of fluctuations implies the useful identity

$$
\begin{aligned}
\left\langle\int_{M} \boldsymbol{w}_{\beta} \cdot \nabla_{\boldsymbol{w}_{\beta}} \boldsymbol{v} \mathrm{d} \boldsymbol{x}\right\rangle & =\int_{M}\left\langle\boldsymbol{w}_{\beta i} \boldsymbol{w}_{\beta k}\right\rangle\left(\frac{\partial v_{i}}{\partial x_{k}}\right) \mathrm{d} \boldsymbol{x} \\
& =\int_{M} \frac{\partial v_{\alpha}}{\partial x_{\alpha}} \mathrm{d} \boldsymbol{x}=\int_{M} \operatorname{div} \boldsymbol{v}_{h} \mathrm{~d} \boldsymbol{x} \\
& =0,
\end{aligned}
$$

where the last equality follows from divergence theorem since $\boldsymbol{v}_{h}$ is tangential to the boundary of $M$.

Define $\Delta^{-1} \operatorname{div} \boldsymbol{v}$ as the solution of Poisson's equation

$$
\Delta \psi=\operatorname{div} \boldsymbol{v} \quad \text { in } M,
$$

with matching Neumann's boundary conditions

$$
\frac{\partial \psi}{\partial z}=v_{3} \text { on } \partial M
$$

Then, from (27),

$$
\phi_{\beta}=-\nabla \Delta^{-1} \operatorname{div}\left(\nabla_{\boldsymbol{w}_{\beta}} \boldsymbol{w}_{\beta}\right) .
$$

Since the operator $\nabla \Delta^{-1}$ div is $L^{2}$-symmetric,

$$
\begin{aligned}
\left\langle\int_{M} \nabla \phi_{\beta} \cdot \boldsymbol{v} \mathrm{d} \boldsymbol{x}\right\rangle & =-\left\langle\int_{M} \nabla_{\boldsymbol{w}_{\beta}} \boldsymbol{w}_{\beta} \cdot \nabla \Delta^{-1} \operatorname{div} \boldsymbol{v} \mathrm{d} \boldsymbol{x}\right\rangle \\
& =\int_{M}\left\langle\boldsymbol{w}_{\beta} \cdot \nabla_{\boldsymbol{w}_{\beta}} \nabla \Delta^{-1} \operatorname{div} \boldsymbol{v}\right\rangle \mathrm{d} \boldsymbol{x} \\
& =0
\end{aligned}
$$

where we used (57) in order to pass to the last line. Therefore, again integrating by parts and using (60),

$$
\begin{aligned}
\left\langle\int_{M} \mathcal{L}_{\boldsymbol{u}} \phi_{\beta} \cdot \boldsymbol{v} \mathrm{d} \boldsymbol{x}\right\rangle & =\left\langle\int_{M}\left(\nabla_{\boldsymbol{u}} \nabla \phi_{\beta}-\nabla_{\nabla \phi_{\beta}} \boldsymbol{u}\right) \cdot \boldsymbol{v} \mathrm{d} \boldsymbol{x}\right\rangle \\
& =-\left\langle\int_{M} \nabla \phi_{\beta} \cdot\left(\nabla_{\boldsymbol{u}} \boldsymbol{v}+(\nabla \boldsymbol{u})^{T} \boldsymbol{v}\right) \mathrm{d} \boldsymbol{x}\right\rangle=0 .
\end{aligned}
$$


Geometric Lagrangian averaged Euler-Boussinesq and primitive equations

Similarly,

$$
\left\langle\int_{M} \nabla_{\boldsymbol{w}_{\beta}} \nabla_{\boldsymbol{w}_{\beta}} \boldsymbol{u} \cdot \boldsymbol{v} \mathrm{d} \boldsymbol{x}\right\rangle=-\int_{M} \nabla \boldsymbol{u} \cdot \nabla \boldsymbol{v} \mathrm{d} \boldsymbol{x}
$$

Combining (56), (61), and (62) yields

$$
\left\langle\int_{M} \boldsymbol{v} \cdot\left(\nabla_{\nabla_{\boldsymbol{w}_{\beta}} \boldsymbol{w}_{\beta}} \boldsymbol{u}-\nabla_{\boldsymbol{w}_{\beta}} \nabla_{\boldsymbol{w}_{\beta}} \boldsymbol{u}-\mathcal{L}_{\boldsymbol{u}} \nabla \phi_{\beta}\right) \mathrm{d} \boldsymbol{x}\right\rangle=\int_{M} \nabla \boldsymbol{u} \cdot \nabla \boldsymbol{v} \mathrm{d} \boldsymbol{x} .
$$

The potential temperature contribution to the $O\left(\varepsilon^{2}\right)$ Lagrangian is

$$
\begin{aligned}
& \left\langle\int_{M} z\left(\nabla_{\nabla \boldsymbol{w}_{\beta}} \boldsymbol{w}_{\beta} \theta+\nabla_{\nabla \phi_{\beta}} \theta+\nabla_{\boldsymbol{w}_{\beta}} \nabla_{\boldsymbol{w}_{\beta}} \theta\right) \mathrm{d} \boldsymbol{x}\right\rangle \\
& =-\left\langle\int_{M} \boldsymbol{w}_{\beta} \cdot \nabla_{\boldsymbol{w}_{\beta}}(z \nabla \theta)-\nabla \phi_{\beta} \cdot(z \nabla \theta)+\operatorname{div}\left(z \boldsymbol{w}_{\beta}\right) \nabla_{\boldsymbol{w}_{\beta}} \theta \mathrm{d} \boldsymbol{x}\right\rangle \\
& =-\int_{M} 0-0+\left\langle w_{\beta 3} \boldsymbol{w}_{\beta} \cdot \nabla \theta\right\rangle \mathrm{d} \boldsymbol{x}=-\int_{M}\left\langle w_{\beta 3} w_{\beta i}\right\rangle \frac{\partial \theta}{\partial x_{i}} \mathrm{~d} \boldsymbol{x}=0,
\end{aligned}
$$

where the third line follows from (57), (60), and $\left\langle w_{\beta 3} w_{\beta i}\right\rangle \equiv 0$.

Inserting (63) with $\boldsymbol{v}=\boldsymbol{u}+\boldsymbol{R}$ and (64) into (55), then discarding $O\left(\varepsilon^{3}\right)$ terms, we find that the averaged EB Lagrangian in reduced form reads

$$
\begin{aligned}
\bar{\ell}(\boldsymbol{u}, \theta) & =\int_{M} \frac{1}{2}|\boldsymbol{u}|^{2}+\boldsymbol{R} \cdot \boldsymbol{u}-z \theta+\frac{\varepsilon^{2}}{2}\left(|\nabla \boldsymbol{u}|^{2}+\nabla \boldsymbol{R} \cdot \nabla \boldsymbol{u}\right) \mathrm{d} \boldsymbol{x} \\
& =\int_{M} \frac{1}{2}|\boldsymbol{u}|^{2}+\tilde{\boldsymbol{R}} \cdot \boldsymbol{u}-z \theta+\frac{\varepsilon^{2}}{2}|\nabla \boldsymbol{u}|^{2} \mathrm{~d} \boldsymbol{x},
\end{aligned}
$$

where $\tilde{\boldsymbol{R}}$ is the effective Coriolis parameter

$$
\tilde{\boldsymbol{R}}=\boldsymbol{R}-\frac{\varepsilon^{2}}{2} \Delta_{h} \boldsymbol{R} .
$$

Equivalently, the averaged EB Lagrangian reads

$$
\bar{L}(\boldsymbol{\eta}, \dot{\boldsymbol{\eta}})=\int_{M} \frac{1}{2}|\dot{\boldsymbol{\eta}}|^{2}+(\tilde{\boldsymbol{R}} \circ \boldsymbol{\eta}) \cdot \dot{\boldsymbol{\eta}}-\theta_{0} \eta_{3} \mathrm{~d} \boldsymbol{a}+\frac{\varepsilon^{2}}{2} \int_{M}\left|\nabla\left(\dot{\boldsymbol{\eta}} \circ \boldsymbol{\eta}^{-1}\right)\right|^{2} \circ \boldsymbol{\eta} \mathrm{d} \boldsymbol{a} .
$$

\subsection{Primitive equations}

Now we derive the averaged Lagrangian $\bar{L}^{P}$ for the primitive equations. The computation proceeds analogously to the Euler-Boussinesq case with EB Lagrangian $L$ replaced by PE Lagrangian (41). We repeat the argument in (45)-(55), noting that $\boldsymbol{R}=\boldsymbol{R}_{h}$ and $u \cdot v=\boldsymbol{u}_{h} \cdot \boldsymbol{v}_{h}=\boldsymbol{u}_{h} \cdot \boldsymbol{v}$ for arbitrary vector fields $\boldsymbol{u}$ and $\boldsymbol{v}$ so that

$$
\begin{aligned}
L_{\varepsilon}^{P} & =\ell^{P}(u, \theta)+\frac{\varepsilon^{2}}{2}\left\langle\int_{M}\left(\boldsymbol{u}_{h}+\boldsymbol{R}\right) \cdot\left(\nabla_{\nabla_{\boldsymbol{w}_{\beta}} \boldsymbol{w}_{\beta}} \boldsymbol{u}-\nabla_{\boldsymbol{w}_{\beta}} \nabla_{\boldsymbol{w}_{\beta}} \boldsymbol{u}-\mathcal{L}_{\boldsymbol{u}} \nabla \phi_{\beta}\right) \mathrm{d} \boldsymbol{x}\right\rangle \\
& -\frac{\varepsilon^{2}}{2}\left\langle\int_{M} z\left(\nabla_{\nabla_{\boldsymbol{w}_{\beta}} \boldsymbol{w}_{\beta}} \theta+\nabla_{\nabla \phi_{\beta}} \theta+\nabla_{\boldsymbol{w}_{\beta}} \nabla_{\boldsymbol{w}_{\beta}} \theta\right) \mathrm{d} \boldsymbol{x}\right\rangle+O\left(\varepsilon^{3}\right) \\
& =\ell^{P}(u, \theta)+\frac{\varepsilon^{2}}{2} \int_{M} \nabla \boldsymbol{u} \cdot\left(\nabla \boldsymbol{u}_{h}+\nabla \boldsymbol{R}\right) \mathrm{d} \boldsymbol{x}+O\left(\varepsilon^{3}\right) \\
& =\ell^{P}(u, \theta)+\frac{\varepsilon^{2}}{2} \int_{M}\left|\nabla \boldsymbol{u}_{h}\right|^{2}-\boldsymbol{u} \cdot \Delta_{h} \boldsymbol{R} \mathrm{d} \boldsymbol{x}+O\left(\varepsilon^{3}\right),
\end{aligned}
$$


where the second equality is due to (63) and (64).

Thus, the averaged PE Lagrangian is

$$
\bar{\ell}^{P}(\boldsymbol{u}, \theta)=\int_{M} \frac{1}{2}|u|^{2}+\tilde{\boldsymbol{R}} \cdot \boldsymbol{u}-z \theta \mathrm{d} \boldsymbol{x}+\frac{\varepsilon^{2}}{2} \int_{M}\left|\nabla \boldsymbol{u}_{h}\right|^{2} \mathrm{~d} \boldsymbol{x},
$$

or, expressed in terms of the flow map,

$$
\bar{L}^{P}(\boldsymbol{\eta}, \dot{\boldsymbol{\eta}})=\int_{M} \frac{1}{2}|\dot{\eta}|^{2}+(\tilde{\boldsymbol{R}} \circ \boldsymbol{\eta}) \cdot \dot{\boldsymbol{\eta}}-\theta_{0} \eta_{3}+\frac{\varepsilon^{2}}{2} \int_{M}\left|\nabla\left(\dot{\eta} \circ \boldsymbol{\eta}^{-1}\right)\right|^{2} \circ \boldsymbol{\eta} \mathrm{d} \boldsymbol{a} .
$$

\section{Horizontally isotropic Lagrangian averaged Euler-Boussinesq equations}

The horizontally isotropic Lagrangian averaged Euler-Boussinesq equations are EulerPoincaré equations for the averaged Lagrangian $\bar{L}$. To shorten notation, we introduce the effective angular velocity,

$$
\tilde{\Omega}=\boldsymbol{\Omega}-\frac{\varepsilon^{2}}{2} \Delta \boldsymbol{\Omega},
$$

and, borrowing the terminology from Holm $[11,12]$, the circulation velocity

$$
\boldsymbol{v}=\boldsymbol{u}-\varepsilon^{2} \Delta_{h} \boldsymbol{u} .
$$

Taking variations of (65) with Lin constraints (36), integrating by parts and discarding full time derivatives which do not contribute to the action, we find

$$
\begin{aligned}
\delta \bar{\ell} & =\int_{M}(\boldsymbol{u}+\tilde{\boldsymbol{R}}) \cdot \delta \boldsymbol{u}-z \delta \theta+\varepsilon^{2} \nabla \boldsymbol{u} \cdot \nabla \delta \boldsymbol{u} \mathrm{d} \boldsymbol{x} \\
& =\int_{M}(\boldsymbol{v}+\tilde{\boldsymbol{R}}) \cdot(\dot{\boldsymbol{w}}+[\boldsymbol{u}, \boldsymbol{w}])+z \nabla \theta \cdot \boldsymbol{w} \mathrm{d} \boldsymbol{x} \\
& =-\int_{M}\left(\dot{\boldsymbol{v}}+\nabla_{\boldsymbol{u}} \boldsymbol{v}+(\nabla \boldsymbol{u})^{T} \boldsymbol{v}+\tilde{\boldsymbol{\Omega}} \times \boldsymbol{u}+\theta \boldsymbol{e}_{z}\right) \cdot \boldsymbol{w} \mathrm{d} \boldsymbol{x}
\end{aligned}
$$

for arbitrary $\boldsymbol{w} \in T_{\mathrm{id}} \mathcal{D}$. Thus, from the Hodge decomposition we obtain the HILAEB momentum equation

$$
\partial_{t} \boldsymbol{v}+\nabla_{\boldsymbol{u}} \boldsymbol{v}+(\nabla \boldsymbol{u})^{T} \boldsymbol{v}+\tilde{\boldsymbol{\Omega}} \times \boldsymbol{u}+\theta \boldsymbol{e}_{z}+\nabla p=0 .
$$

The same result could be derived by inserting the variational derivatives

$$
\boldsymbol{m}^{\sharp}=\left(\frac{\delta \bar{\ell}}{\delta \boldsymbol{u}}\right)^{\sharp}=\overline{\boldsymbol{R}}+\boldsymbol{v} \quad \text { and } \quad \frac{\delta \ell}{\delta \theta}=-z,
$$

into the abstract Euler-Poincaré equation (38), which yields

$$
\partial_{t} \boldsymbol{v}+(\nabla \times(\boldsymbol{v}+\tilde{\boldsymbol{R}})) \times \boldsymbol{u}-z \nabla \theta+\nabla p=0 .
$$

After redefining the pressure $p \rightarrow p-z \theta-\boldsymbol{u} \cdot \boldsymbol{v},(76)$ simplifies to (74) due to the general vector identity

$$
(\nabla \times \boldsymbol{v}) \times \boldsymbol{u}=\nabla_{\boldsymbol{u}} \boldsymbol{v}-(\nabla \boldsymbol{v})^{T} \boldsymbol{u}=\nabla_{\boldsymbol{u}} \boldsymbol{v}+(\nabla \boldsymbol{u})^{T} v-\nabla(\boldsymbol{u} \cdot \boldsymbol{v}) .
$$

The full set of HILAEB model equations consists of the momentum equation (74), the advection of potential temperature (10b), and the incompressibility constraint (10c) combined with no-mass-flux boundary conditions (11). 
As a Hamiltonian model, HILAEB conserve the energy

$$
\begin{aligned}
E(\boldsymbol{u}, \theta) & =\int_{M} \boldsymbol{m} \cdot \boldsymbol{u} \mathrm{d} \boldsymbol{x}-\bar{\ell}(\boldsymbol{u}, \theta) \\
& =\int_{M} \frac{1}{2}|\boldsymbol{u}|^{2}+\varepsilon^{2}|\nabla \boldsymbol{u}|^{2}+\theta z \mathrm{~d} \boldsymbol{x} .
\end{aligned}
$$

The invariance of the Lagrangian $\bar{L}$ under particle relabelling implies the existence of materially conserved PV,

$$
q=\left(\nabla \times \boldsymbol{m}^{\sharp}\right) \cdot \nabla \theta=(\tilde{\boldsymbol{\Omega}} \times \boldsymbol{u}+\nabla \times \boldsymbol{v}) \cdot \nabla \theta .
$$

The simplest way to derive the expression for PV (79) is to take the wedge product of the abstract EP equation (37) on the space of one-forms with $\mathrm{d} \theta$. We refer the reader to [23] for the details. Alternatively, one obtains the same result after a rather tedious computation by taking the inner product of $\nabla \theta$ with the curl of (74).

We remark that PV form (79) is identical to the original Euler-Boussinesq system, with $\boldsymbol{v}$ replacing fluid velocity $\boldsymbol{u}$ under the curl operator. This justifies the use of "circulation velocity" term for $\boldsymbol{v}$, as it is the quantity entering Kelvin's circulation theorem corresponding to material conservation of $q$, namely,

$$
\frac{\mathrm{d}}{\mathrm{d} t} \oint_{\gamma(t)}(\boldsymbol{v}+\tilde{\boldsymbol{R}}) \cdot \mathrm{d} \boldsymbol{x}=-\oint_{\gamma(t)} \theta \mathrm{d} \boldsymbol{x},
$$

whenever the curve $\gamma(t)$ moves with the fluid.

\section{Horizontally isotropic Lagrangian averaged primitive equations}

To obtain horizontally isotropic Lagrangian averaged primitive equations (HILAPE), we proceed as in (73). Computing the variation of the averaged PE Lagrangian (69) yields

$$
\begin{aligned}
\delta \bar{\ell}^{P} & =\int_{M}(\boldsymbol{v}+\tilde{\boldsymbol{R}}) \cdot(\dot{\boldsymbol{w}}+[\boldsymbol{u}, \boldsymbol{w}])+z \nabla \theta \cdot \boldsymbol{w} \mathrm{d} \boldsymbol{x} \\
& =-\int_{M}\left(\dot{\boldsymbol{v}}+\nabla_{\boldsymbol{u}} \boldsymbol{v}+\left(\nabla \boldsymbol{u}_{h}\right)^{T} \boldsymbol{v}+\tilde{f} \boldsymbol{u}^{\perp}+\theta \boldsymbol{e}_{z}\right) \cdot \boldsymbol{w} \mathrm{d} \boldsymbol{x}
\end{aligned}
$$

for arbitrary vector field $\boldsymbol{w} \in \mathfrak{X}_{\text {div }}^{s}(M)$, where

$$
\boldsymbol{v}=\boldsymbol{u}_{h}-\varepsilon^{2} \Delta_{h} \boldsymbol{u}_{h} \quad \text { and } \quad \tilde{f}=f-\frac{\varepsilon^{2}}{2} \Delta_{h} f
$$

are the circulation velocity and the effective Coriolis parameter, respectively. Therefore, the HILAPE momentum equation is given by

$$
\partial_{t} \boldsymbol{v}+\nabla_{\boldsymbol{u}} \boldsymbol{v}+\left(\nabla \boldsymbol{u}_{h}\right)^{T} \boldsymbol{v}+\tilde{f} \boldsymbol{u}^{\perp}+\theta \boldsymbol{e}_{z}+\nabla p=0 .
$$

Naturally, computing the Euler-Poincaré equations via (37) with

$$
\boldsymbol{m}^{\sharp}=\left(\frac{\delta \ell}{\delta \boldsymbol{u}}\right)^{\sharp}=\boldsymbol{u}_{h}-\varepsilon^{2} \Delta \boldsymbol{u}_{h}+\tilde{\boldsymbol{R}} \quad \text { and } \frac{\delta \ell}{\delta \theta}=-z
$$

yields the same result. 
Similar to the Euler-Boussinesq case, the full set of HILAPE consists of the momentum equation (83) with no-mass-flux boundary conditions (11), the advection of potential temperature $(12 b)$, and the incompressibility constraint $(12 c)$.

As an Euler-Poincaré system, HILAPE conserve the energy

$$
E(\boldsymbol{u}, \theta)=\int_{M} \frac{1}{2}\left(|u|^{2}+\varepsilon^{2}|\nabla u|^{2}\right)+\theta z \mathrm{~d} x
$$

and the potential vorticity $q$ on fluid particles,

$$
q=\left(\nabla \times \boldsymbol{m}^{\sharp}\right) \cdot \nabla \theta=(\tilde{f}+\nabla \times \boldsymbol{v}) \cdot \nabla \theta,
$$

with corresponding Kelvin's circulation theorem

$$
\frac{d}{d t} \oint_{\gamma(t)}(\boldsymbol{v}+\tilde{\boldsymbol{R}}) \cdot \mathrm{d} \boldsymbol{x}=-\oint_{\gamma(t)} \theta \mathrm{d} \boldsymbol{x}
$$

for an arbitrary closed curve $\gamma(t)$ moving with fluid velocity.

\section{Discussion}

Inspired by the work by Gilbert and Vanneste [10] and Oliver [22], we have derived the analogues of EB- $\alpha$ and PE- $\alpha$ via geometric Lagrangian averaging.

In order to derive the HILAEB and HILAPE models, we have replaced the 3D isotropy with horizontal one (28b) which is consistent with no-mass-flux on vertical boundaries. An alternative approach leads to anisotropic equations, similar to Lagrangian mean Euler-Boussinesq [11] and anisotropic Lagrangian averaged Euler [21], which introduce additional dynamics for the mean covariance tensor $\langle\boldsymbol{w} \otimes \boldsymbol{w}\rangle$. HILAEB and HILAPE differ from EB- $\alpha$

$$
\begin{aligned}
& \partial_{t} \boldsymbol{v}+\nabla_{\boldsymbol{u}} \boldsymbol{v}+(\nabla \boldsymbol{u})^{T} \boldsymbol{v}+\boldsymbol{\Omega} \times \boldsymbol{u}+\theta \boldsymbol{e}_{z}+\nabla p=0, \\
& \boldsymbol{v}=\boldsymbol{u}-\varepsilon^{2} \Delta \boldsymbol{u},
\end{aligned}
$$

and PE- $\alpha$

$$
\begin{aligned}
& \partial_{t} \boldsymbol{v}+\nabla_{\boldsymbol{u}} \boldsymbol{v}+\left(\nabla \boldsymbol{u}_{h}\right)^{T} \boldsymbol{v}+f \boldsymbol{u}^{\perp}+\theta \boldsymbol{e}_{z}+\nabla p=0 . \\
& \boldsymbol{v}=\boldsymbol{u}_{h}-\varepsilon^{2} \Delta \boldsymbol{u}_{h},
\end{aligned}
$$

respectively, in the Coriolis terms and in the Laplacian operator defining the circulation velocity $\boldsymbol{v}$.

The full 3D Laplace operator appearing in EB- $\alpha$ and $\mathrm{PE}-\alpha$ requires additional boundary conditions which do not arise naturally. Usually either no-slip or Navierslip conditions are imposed, see[19, 30]. HILAEB and HILAPE replace the Helmoltz operator defining the circulation velocity in $\mathrm{EB}-\alpha$ and $\mathrm{PE}-\alpha$, respectively, by a horizontal one, which can be inverted on $M$ without imposing additional ad hoc boundary conditions. In contrast to the $\mathrm{EB}-\alpha$ and PE- $\alpha$ equations, the filtering provided by horizontal Laplacian has no effect on vertical waves.

It should be noted that while the difference from HILAEB and HILAPE from EB- $\alpha$ in the Coriolis term is insignificant and vanishes on an $f$ - or $\beta$-plane, the difference in the Laplace operator defining the circulation velocity $\boldsymbol{v}$ needs to be examined in more detail. 


\section{Acknoledgements}

We thank anonymous reviewers for helpful suggestions and encouragement. This work

is a contribution to the Collaborative Research Centre TRR 181 "Energy Transfer in Atmosphere and Ocean" funded by the German Research Foundation.

\section{References}

[1] Aizinger V, Korn P, Giorgetta M and Reich S 2015 Large-scale turbulence modelling via $\alpha$ regularisation for atmospheric simulations, J. Turbul. 16 367-391

[2] Andrews D G and McIntyre M E 1978 An exact theory of nonlinear waves on a Lagrangian-mean flow J. Fluid Mech. 89 609-646

[3] Andrews D G and McIntyre M E 1978 On wave-action and its relatives J. Fluid Mech. 89 647-664

[4] Arnold V I 1966 Sur la géometrie différentielle des groupes de Lie de dimension infinie et ses applications à l'hydrodynamique des fluides parfaits Ann. Inst. Fourier 16 319-361

[5] Badin G and Crisciani F 2018 Variational Formulation of Fluid and Geophysical Fluid Dynamics: Mechanics, Symmetries and Conservation Laws (Springer)

[6] Bühler O 2014 Waves and Mean Flows (Cambridge: Cambridge University Press)

[7] Chen S, Foias C, Holm D D, Olson E, Titi E S and Wynne S 1999 A connection between the Camassa-Holm equations and turbulent flows in channels and pipes Phys. Fluids 11 2343-2353

[8] Ebin D G and Marsden J E 1970 Groups of diffeomorphisms and the motion of an incompressible fluid Ann. Math. 92 102-163

[9] Franzke C L E, Oliver M, Rademacher J D M and Badin G 2018 Multi-scale methods for geophysical flows (submitted for publication)

[10] Gilbert A D and Vanneste J 2018 Geometric generalised Lagrangian-mean theories J. Fluid Mech. 839 95-134

[11] Holm D D 1999 Fluctuation effects on 3D Lagrangian mean and Eulerian mean fluid motion Physica D 133 215-269

[12] Holm D D 2002 Lagrangian averages, averaged Lagrangians, and the mean effects of fluctuations in fluid dynamics Chaos 12 518-530

[13] Holm D D 2002 Averaged Lagrangians and the mean effects of fluctuations in ideal fluid dynamics Physica D $\mathbf{1 7 0} 253-286$

[14] Holm D D, Jeffery C, Kurien S, Livescu D, Taylor M A and Wingate B A 2005 The LANS- $\alpha$ model for computing turbulence: Origins, results, and open problems, Los Alamos Science 29 152-172

[15] Holm D D, Marsden J E and Ratiu T S 1998 The Euler-Poincarè Equations and Semidirect Products with Applications to Continuum Theories, Adv. Math. 137 1-81.

[16] Holm D D, Marsden J E and Ratiu T S 2002 The Euler-Poincarè equations in geophysical fluid dynamics, Large-Scale Atmosphere-Ocean Dynamics vol 1 (Cambridge: Cambridge University Press) pp 251-300

[17] Holm D D, Schmah T and Stoica C 2009 Geometric Mechanics and Symmetry: from Finite to Infinite dimensions (Oxford University Press)

[18] Petersen M R, Hecht M W, Holm D D and Wingate B A 2008 Implementation of the LANS- $\alpha$ turbulence model in a primitive equation ocean model, J. Comp. Phys. 227 5691-5716

[19] Lopes Filho M C, Nussenzveig-Lopes H J, Titi E S and Zang A, 2015 Convergence of the 2D Euler- $\alpha$ to Euler equations in the Dirichlet case: Indifference to boundary layers, Physica $D$ 292-293 51-61

[20] Porta Mana P and Zanna L 2014 Toward a stochastic parameterization of ocean mesoscale eddies Ocean Model. 79 1-20

[21] Marsden J E and Shkoller S 2003 The anisotropic Lagrangian averaged Euler and Navier-Stokes equations Arch. Rat. Mech. and Analysis 166 27-46 
[22] Oliver M 2017 Lagrangian averaging with geodesic mean Proc. Royal Soc. 473 doi:10.1098/rspa.2017.0558.

[23] Oliver M and Vasylkevych S 2016 Generalized large-scale semigeostrophic approximations for the $f$-plane primitive equations J. Phys. A 49184001

[24] Oliver M and Vasylkevych S 2018 Geodesic motion on the groups of diffeomorphisms with $H^{1}$ metric as geometric generalised Lagrangian mean theory in preparation

[25] Palais R S 1968 Foundations of global nonlinear analysis (New York: Benjamin)

[26] Roberts P H and Soward A M 2006 Eulerian-Lagrangian means in rotating magnetohydrodynamic flows I. General results Geophys. Astrophys. Fluid Dynam. 100 457-483

[27] Roberts P H and Soward A M 2006 Covariant description of non-relativistic magnetohydrodynamics Geophys. Astrophys. Fluid Dynam. 100 485-502

[28] Salmon R 2013 An alternative view of generalized Lagrangian mean theory. J. Fluid Mech. 719 $165-182$

[29] Salmon R 2016 Variational treatment of inertia-gravity waves interacting with a quasi-geostrophic mean flow J. Fluid Mech. 809 502-529

[30] Shkoller S 2000 Analysis on groups of diffeomorphisms of manifolds with boundary and the averaged motion of a fluid J. Diff. Geom. 55 145-191.

[31] Shkoller S 2002 The Lagrangian averaged Euler (LAE- $\alpha$ ) equations with free-slip or mixed boundary conditions, Geometry, Mechanics, and Dynamics ed P Newton, P Holmes et al (New York: Springer)

[32] Soward A M and Roberts P H 2010 The hybrid Euler-Lagrange procedure using an extension of Moffatt's method J. Fluid Mech. 661 45-72

[33] Soward A M and Roberts P H 2014 Eulerian and Lagrangian means in rotating, magnetohydrodynamic flows II. Braginsky's nearly axisymmetric dynamo Geophys. Astrophys. Fluid Dynam. 108 269-322

[34] Wagner G L and Young W R 2015 Available potential vorticity and wave-averaged quasigeostrophic flow J. Fluid Mech. 785 401-424

[35] Xie J-H and Vanneste J 2015 A generalised-Lagrangian-mean model of the interactions between near-inertial waves and mean flow J. Fluid Mech. 774 143-169 